\title{
Granzyme B for predicting the durable clinical benefit of anti- PD-1/PD-L1 immunotherapy in patients with non-small cell lung cancer
}

\author{
Jae Heun Chung ${ }^{1, \#}$, Jong Seong Ha ${ }^{3,4 \#}$, Jaewoo Choi ${ }^{3}$, Sang Mo Kwon ${ }^{3,4}$, Mi Sook Yun ${ }^{2}$, Taehwa Kim ${ }^{1,2}$, \\ Doosoo Jeon $^{1,2}$, Seong Hoon Yoon ${ }^{1,2}$, Yun Seong Kim ${ }^{1,2}$ \\ ${ }^{1}$ Department of Internal Medicine, College of Medicine, Pusan National University, Pusan National University Yangsan Hospital, Yangsan, South \\ Korea; ${ }^{2}$ Research Institute of Convergence Biomedical Science and Technology, Pusan National University Yangsan Hospital, Yangsan, South Korea; \\ ${ }^{3}$ Laboratory of Regenerative Medicine and Stem Cell Biology, Department of Physiology, Medical Research Institute, School of Medicine, Pusan \\ National University, Yangsan, South Korea; ${ }^{4}$ BK21 GRAND Convergence Medical Science Eduation Rsearch Center, Pusan National University, \\ Yangsan, South Korea \\ Contributions: (I) Conception and design: JH Chung, SH Yoon, YS Kim; (II) Administrative support: None; (III) Provision of study materials or \\ patients: JH Chung, JS Ha, J Choi, T Kim; (IV) Collection and assembly of data: J Choi, JS Ha; (V) Data analysis and interpretations: JH Chung, SH \\ Yoon, D Jeon, JS Ha, MS Yun, SM Kwon; (VI) Manuscript writing: All authors; (VII) Final approval of manuscript: All authors. \\ \#These authors contributed equally to this work. \\ Correspondence to: Seong Hoon Yoon, MD; Yun Seong Kim, MD, PhD. Pusan National University Yangsan Hospital, Geumo-ro 20, Beomeo-ri, \\ Mulgeum-eup, Yangsan-si, Gyeongsangnam-do 626-770, Korea. Email: drysh79@gmail.com; yskim@pusan.ac.kr.
}

Background: To identify immunotherapy biomarkers, we examined granzyme B levels in peripheral blood $\mathrm{PD}-1^{+} \mathrm{CD}^{+} \mathrm{T}$ cells and their relationship with treatment outcomes in patients with non-small cell lung cancer (NSCLC).

Methods: To evaluate the association of granzyme B with response to immunotherapy, we tested blood samples obtained from 16 patients with stage IIIC to IV NSCLC receiving immune-checkpoint inhibitor treatment. We used flow cytometry to measure the change in the percentage of $\mathrm{PD} 1^{+} \mathrm{CD} 8^{+} \mathrm{T}$ cells expressing granzyme B before ( $\mathrm{t} 0$ ) and after ( $\mathrm{t} 1$ ) immunotherapy, and we evaluated for an association with tumor response to therapy, progression-free survival (PFS) and overall survival (OS). Additionally, we measured immune markers correlated with immunotherapy response by enzyme-linked immunosorbent assay.

Results: We found that the sequential change of granzyme $\mathrm{B}+\mathrm{T}$ cells after immunotherapy (t1/t0) significantly predicted durable clinical benefit (DCB) compared to no clinical benefit (NCB) $(\mathrm{P}=0.048)$, and prolonged PFS $(\mathrm{P}=0.025)$. Patients who demonstrated a PD-L1 tumor proportion score (TPS) $>50 \%$ showed NCB if patients had low granzyme B t1/t0 levels $(<0.805)$. Additionally, all patients with $1 \%$ PD-L1 TPS (or higher) and high granzyme B $\mathrm{t} 1 / \mathrm{t} 0$ ( $\geq 0.805)$ showed DCB. Therefore, granzyme B t1/t0 may be an adjunctive marker with available PD-L1 TPS.

Conclusions: Our findings revealed that sequential change in granzyme B might be utilized as a predictive biomarker of immune checkpoint inhibitor monotherapy.

Keywords: Granzyme B; programmed death-1; CD8 T cells; immunotherapy; non-small cell lung cancer (NSCLC)

Submitted Nov 12, 2021. Accepted for publication Jan 19, 2022.

doi: $10.21037 /$ tcr-21-2506

View this article at: https://dx.doi.org/10.21037/tcr-21-2506 


\section{Introduction}

Despite a steadily dropping death rate, lung cancer remains the leading cause of cancer-related mortality worldwide (1). Most patients visiting clinics are diagnosed at an advanced stage owing to the absence of self-recognized respiratory symptoms. These patients thus experience unsatisfactory clinical outcomes with limited cytotoxic chemotherapy efficacy. Programmed cell death (PD)-1/PD ligand (PD-L1) inhibitors have recently demonstrated antitumor effects in advanced and metastatic non-small cell lung cancer (NSCLC) patients and have therefore been approved by the United States Food and Drug Administration as standard therapy (2). The tumor proportion score (TPS) of PD-L1 has been used as a predictive marker for immunotherapeutic agents (3-5). For example, one clinical trial demonstrated that a PD-L1 TPS level of $50 \%$ or higher improved the patient survival rate to a greater extent than conventional chemotherapy (6). However, certain trials yielded controversial results concerning the relationship between the PD-L1 TPS level and its predictive power for survival (7-9).

Regardless of PD-L1 TPS level, PD-1/PD-L1 blockade in patients with advanced NSCLC can accelerate tumor growth (10). Therefore, researchers working in translational research have focused on developing other biomarkers, including microbiome profiles (11) and tumor mutation burdens (12). However, to date, none of these have shown sufficient predictive value for use in real-world clinical settings. Interestingly, a change in the percentage of peripheral blood $\mathrm{PD}-1^{+} \mathrm{CD} 8^{+} \mathrm{T}$ cells after immunotherapy is a predictor of anti-PD-1 therapy prognosis in solid tumors (13). Cytotoxic $\mathrm{CD}^{+} \mathrm{T}$ cells mount immune responses against cancer cells by releasing cytolytic granules containing granzyme B (GrmB) and cytokines.

A previous study presented evidence that these mediators induce cell death resistance essential for the survival and proliferation of lung cancer cells (14). Among cytolytic granules, GrmB-detected using nanoparticles and visualized with positron emission tomographycomputed tomography-reportedly predicts response to immunotherapy (15). However, changes in the percentage of peripheral blood PD $-1^{+} \mathrm{CD} 8^{+} \mathrm{T}$ cells expressing GrmB, and any correlation with clinical outcomes, remain to be elucidated. Therefore, this study investigated whether the percentage of peripheral blood $\mathrm{PD}-1^{+} \mathrm{CD} 8^{+} \mathrm{T}$ cells expressing GrmB in patients with advanced NSCLC could predict treatment outcomes. We also investigated other biomarkers that correlate with immunotherapy response, including PD-L1 TPS, and expression of cytokinesinterleukin-2 (IL-2), tumor necrosis factor-alpha (TNF $\alpha$ ), and interferon-gamma (IFN- $\gamma$ ).

We present the following article in accordance with the REMARK reporting checklist (available at https://tcr. amegroups.com/article/view/10.21037/tcr-21-2506/rc).

\section{Methods}

\section{Patients}

Our study enrolled patients with histologically confirmed advanced NSCLC (IIIC to IV) who were deemed clinically unfit for surgery, according to the Union for International Cancer Control-TNM classification (8th edition). Patients manifested at least one measurable lesion that had progressed after two cycles of a platinumbased chemotherapy regimen without known mutations in epidermal growth factor receptor activation or anaplastic lymphoma receptor tyrosine kinase translocation. All patients exhibited an Eastern Cooperative Oncology Group performance status of $0-1$. Individuals who visited for a general health check-up were recruited as normal healthy controls. The study was conducted in accordance with the Declaration of Helsinki (as revised in 2013). It was approved by the Institutional Review Board of Pusan National University Yangsan Hospital (IRB 05-2018-061) and informed consent was taken from all the patients.

\section{Study design and treatments}

After platinum doublet chemotherapy, all patients received immune checkpoint inhibitors as their second-line treatment. The patient received an intravenous infusion of pembrolizumab (200 $\mathrm{mg}$ every three weeks), nivolumab ( $2 \mathrm{mg} / \mathrm{kg}$ every two weeks), or atezolizumab $(1,200 \mathrm{mg}$ every three weeks). Treatment continued until radiological progression, unacceptable toxic effect manifestation, or withdrawal of patient consent. Computed tomography was conducted every six weeks. Peripheral blood was collected from all 16 patients within two weeks before treatment [baseline, treatment $0(\mathrm{t} 0)$ ] and approximately three weeks after the first dose of immunotherapy [treatment 1 (t1)]. Tumor response was assessed by chest computed tomography every six weeks according to the Response Evaluation Criteria In Solid Tumors (RECIST), version 1.1. Durable clinical benefit (DCB) was defined as a complete response (CR), partial response (PR), or stable disease (SD) 
lasting longer than 4.5 months. No clinical benefit (NCB) was defined as progressive disease (PD). Progression-free survival (PFS) and overall survival (OS) were considered the primary endpoint and defined as the duration from the start of immunotherapy to either disease progression or death from any cause. The average sampling time was 21 days (range 18-25 days) (Table S1).

\section{Flow cytometry}

We isolated human peripheral blood mononuclear cells (PBMCs) from whole blood collected in K3 ethylenediamine-tetraacetic acid (EDTA) vacutainer tubes with a total of $9 \mathrm{~mL}$ using an Eppendorf 5702R centrifuge (Hamburg, Germany). Cells were centrifuged at $400 \times \mathrm{g}$ and $4{ }^{\circ} \mathrm{C}$ for five minutes. The pellet was resuspended in $100 \mu \mathrm{L}$ of $2 \mathrm{mM}$ EDTA/phosphate-buffered saline and stained with forkhead box protein P3 (FoxP3), T cell immunoglobulin and mucin domain-containing-3 (Tim-3), lymphocyte activation gene-3 (LAG-3), and cytotoxic T lymphocyte antigen-4 (CTLA-4) antibodies (Table S2) for one hour at $4{ }^{\circ} \mathrm{C}$. Samples were subjected to flow cytometry using Fluorescence-activated cell sorting (FACS) Aria III (BD Biosciences, Franklin 5 Lakes, NJ, USA). Data were analyzed in FlowJo software (BD Biosciences). Gating strategies are summarized in Figure S1.

\section{T-cell sorting and in vitro expansion of $C D 8^{+} T$ cells}

For isolation of cell subsets, cells were thawed, washed, and resuspended in $500 \mu \mathrm{L}$ of staining buffer containing antibodies. After 30 minutes of incubation at $4{ }^{\circ} \mathrm{C}$, cells were washed twice and sorted. Cells were gated on live 7-aminoactinomycin D-positive/propidium iodide-negative single cells, $\mathrm{CD}^{+}$cells, and the population of interest. One sample consisted of four cell types: GrmB $(-,+)$ and PD-1 $(-,+) \mathrm{T}$ cells. From the cells of 16 samples, $5 \times 10^{3} \mathrm{~T}$ cells were expanded in T cell medium (1:1 mix of AIMV (Thermo Fisher, Waltham, MA, USA) and RPMI 1640 (SigmaAldrich, St. Louis, MO, USA) medium, 5\% human serum (mix of three donors, Blutspendezentrum Basel), 100 units penicillin, $100 \mu \mathrm{g} / \mathrm{mL}$ streptomycin, $2 \mathrm{mM} \mathrm{L}$-glutamine, and $25 \mathrm{mM} \mathrm{HEPES}$ ), supplemented with 3,000 IU of IL-2 (Peprotech, Rocky Hill, NJ, USA) and $30 \mathrm{ng} / \mathrm{mL}$ anti-CD3 (OKT3, Biolegend, San Diego, CA, USA). After day 2, half of the medium was replaced with a fresh medium containing IL-2 every two days. On day 8, T cells were tested for PD-1 expression and IL-2, TNF- $\alpha$, and IFN- $\gamma$ release.

\section{Enzyme-linked immunosorbent assays (ELISAs)}

We collected $\mathrm{CD} 8^{+} \mathrm{T}$-cell culture supernatants from 24-well plates. We determined proinflammatory factors production using ELISA kits specific for IL-2, TNF- $\alpha$, and IFN- $\gamma$, following the manufacturer's protocol (Abcam, Cambridge, MA, USA) and then normalized to cell protein concentrations.

\section{PD-L1 immunobistochemical staining assay}

Immunohistochemical (IHC) staining for PD-L1 (SP263) was performed on a Ventana Benchmark automated staining platform (Ventana Medical Systems, Inc., Tucson, AZ, USA) using a VENTANA OptiView diaminobenzidine tetrahydrochloride IHC Detection Kit (P/N 760-700) and its staining protocol. The formalin-fixed paraffinembedded tissues of all cases were cut, dried, deparaffinized, rehydrated, and heated following the protocol. PD-L1 expression was categorized according to TPS into "high" (if at least $50 \%$ of the tumor cells stained positive) and "low" (if $0-49 \%$ of the tumor cells stained negative). Two independent, board-certified pathologists interpreted IHC staining. No discordance between the two pathologists was observed.

\section{Statistical analyses}

The non-parametric Mann-Whitney U test compared DCB and NCB based on all parameters, including GrmB $\mathrm{t} 1 / \mathrm{t} 0$ levels. A receiver operating characteristic (ROC) curve was calculated, and a 6 area under the curve of $>0.8$ was used for improved predictive power. The analysis was also used to determine the optimal cutoff value, then used with the Kaplan-Meier method to estimate PFS. To assess concordance between PD-L1 TPS and GrmB t1/t0, we calculated intraclass correlation coefficients (ICCs). The ICCs were interpreted as follows: $<0.05$. Statistical analyses were performed using SPSS version 21 (SPSS, version 21.0; IBM Corp., Armonk, NY, USA).

\section{Results}

\section{Patient characteristics}

To investigate GrmB's association with immune checkpoint inhibitor response, we prospectively collected data and blood samples from patients with advanced NSCLC. We initially enrolled 25 patients in this study. However, 
Table 1 Patient characteristics

\begin{tabular}{lcc}
\hline Characteristics & DCB $(\mathrm{n}=10)$ & NCB $(\mathrm{n}=6)$ \\
\hline Age (years), median [range] & $72[50-85]$ & $71.5[46-87]$ \\
Gender, male & $10(100 \%)$ & $6(100 \%)$ \\
Histology & & \\
Non-squamous & $3(30 \%)$ & $3(50 \%)$ \\
Squamous & $7(70 \%)$ & $3(50 \%)$ \\
Smoking status & & \\
Current & $5(50 \%)$ & $3(50 \%)$ \\
Former & $5(50 \%)$ & $3(50 \%)$ \\
Stage & & \\
IIIC & $2(20 \%)$ & $1(17 \%)$ \\
IV & $8(80 \%)$ & $5(83 \%)$ \\
PD-L1 expression & & \\
$0 \%$ & $1(10 \%)$ & $2(33 \%)$ \\
$1-50 \%$ & $3(30 \%)$ & $1(17 \%)$ \\
$\geq 50 \%$ & $6(60 \%)$ & $3(50 \%)$ \\
\hline
\end{tabular}

DCB, durable clinical benefit; NCB, no clinical benefit; PD-L1, programmed cell death ligand-1.

nine patients were excluded for not meeting the inclusion criteria regarding lasting response period. All 16 patients had received previous platinum-based combination chemotherapy before immunotherapy and had not undergone treatment with immune-checkpoint inhibitors before this experiment. All patients with median age of 72 years (range 46-87 years) were male and were current smokers or former smokers. There was no significant difference of histologic types, TNM stage, and PD-L1 expression between two groups. Patients exhibited good performance scores $(0-1)$. Of the 16 patients, $10(62.5 \%)$ achieved a durable clinical benefit, as per RECIST v1.1, and the remaining $6(37.5 \%)$ patients showed no durable benefit. PFS and OS differs significantly between the two groups [median $\mathrm{PFS}, 11.40$ vs. 1.75 months $(\mathrm{P}<0.0001)$; median OS, 28.20 vs. 10.85 months $(\mathrm{P}=0.013)$, Figure S2]. The patient characteristics for each response group are summarized in Table 1.

\section{Peripheral blood $\mathrm{PD}-\mathrm{1}^{+} \mathrm{CD} 8^{+} \mathrm{T}$ cells did not show a proliferative response after immunotherapy}

To assess the role of $\mathrm{PD}-1^{+} \mathrm{CD} 8^{+} \mathrm{T}$ cells as a predictive immunotherapy marker, we examined the PD-1 expression fold-change in live $\mathrm{CD} 8^{+} \mathrm{T}$ cells $(\mathrm{t} 1 / \mathrm{t} 0)$ from peripheral blood of the same patients before and after immunotherapy (Figure S3). Patients with DCB and with NCB did not differ significantly in percentage of $\mathrm{PD}-1^{+} \mathrm{CD} 8^{+} \mathrm{T}$ cells (t1/ t0) (DCB; 9.45 vs. $8.58, \mathrm{P}=0.958$; Figure $1 A$ ). This analysis also failed to show an association of PFS and OS with immunotherapy $(\mathrm{P}=0.21$, Figure $1 B ; \mathrm{P}=0.53$, Figure $1 C)$.

\section{Intracellular GrmB $11 /$ t0 of peripheral blood PD- $1^{+} C D 8^{+}$ $T$ cells predicted tumor response to immunotherapy and clinical outcomes in patients with NSCLC}

We sorted T cells to study the role of GrmB t1/ t0 in peripheral blood $\mathrm{PD}-1^{+} \mathrm{CD} 8^{+} \mathrm{T}$ cells during immunotherapy. We examined PBMCs from 16 patients with NSCLC and normal healthy controls and found that the percentage of $\mathrm{GrmB}^{+} \mathrm{PD}-1^{+} \mathrm{CD} 8^{+} \mathrm{T}$ cells increased after immunotherapy in five of the 10 patients with $\mathrm{DCB}$ and slightly decreased without significance in the remaining five. On the other hand, the percentage of $\mathrm{GrmB}^{+} \mathrm{PD}$ $1^{+} \mathrm{CD} 8^{+} \mathrm{T}$ cells significantly decreased in five of the six patients with NCB after administration of immunotherapy (Figure S4).

Based on these data, we found a significant difference in GrmB t1/t0 between patients with DCB and patients with NCB. Patients with DCB had significantly higher GrmB t1/ t0 than patients with $\mathrm{NCB}$ (mean of 1.75 vs. $0.52, \mathrm{P}=0.048$; Figure $2 A$ ). Furthermore, GrmB t1/t0 demonstrated a high predictive value for DCB (area under the curve $=0.85, \mathrm{P}=0.022 ;$ Figure $2 B$ ). An optimal cutoff of 0.805 for GrmB $\mathrm{t} 1 / \mathrm{t} 0$ was determined based on the ROC curve. The sensitivity and specificity of this cutoff were $80.0 \%$ and $83.3 \%$, respectively. The probability of DCB was higher in patients with $\mathrm{GrmB} \mathrm{t} 1 / \mathrm{t} 0 \geq 0.805$ than in patients with GrmB t1/t0<0.805 (80.0 vs. 20.0\%, $\mathrm{P}<0.05$; Figure $2 C$ ). Patients with GrmB t1/t0 fold-changes above the cutoff had a median PFS of 9.4 months (range 2.3-20.6 months) versus a median PFS of 2.4 months (range 1.5-10.4 months) for patients with low $\mathrm{GrmB}$ fold-changes $(\mathrm{P}=0.025$ for PFS comparison; Figure 2D). We also examined the clinical significance in OS, based on the cutoff value of GrmB $\mathrm{t} 1 / \mathrm{t} 0$. Patients with high sequential changes in $\mathrm{GrmB}$ had a median survival of 24.6 months, while those with low sequential changes in GrmB had that of 9.2 months. Although two groups in terms of $\mathrm{GrmB} \mathrm{t} 1 / \mathrm{t} 0$ classified by cutoff value had well separated OS line, results did not fit statistical significance between two groups $(\mathrm{P}=0.18$ for $\mathrm{OS}$ 

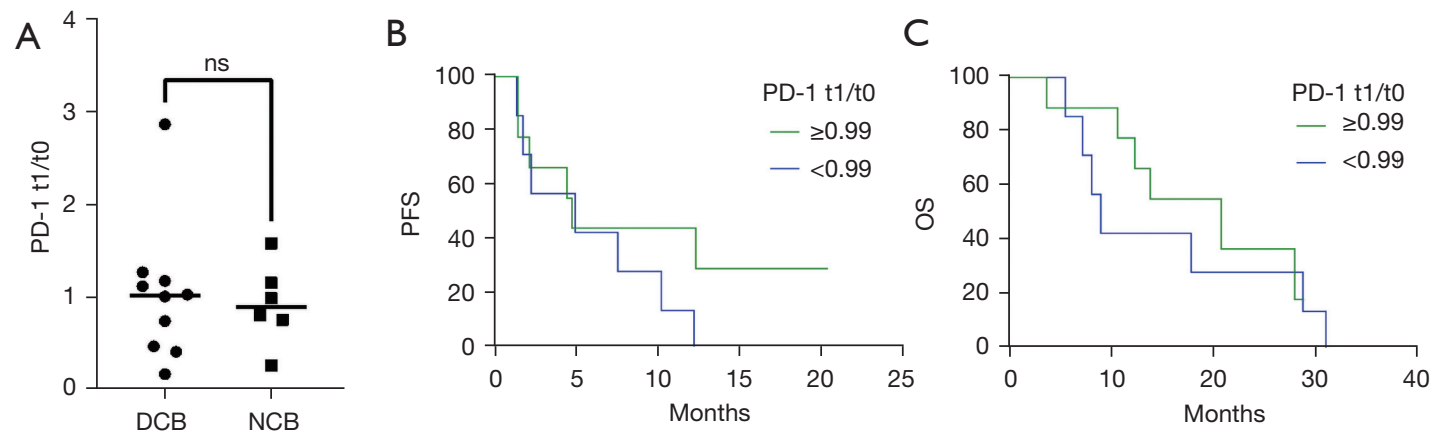

Figure 1 Analysis of the sequential change in peripheral blood live PD- $1^{+} \mathrm{CD} 8^{+} \mathrm{T}$ cells associated with the immune-checkpoint inhibitor response. (A) Comparison of PD- $1^{+} \mathrm{CD}^{+} \mathrm{T}$ cells $\mathrm{t} 1 / \mathrm{t} 0$ between $\mathrm{DCB}$ and NCB subgroups after PD-1/PD-L1 inhibitor treatment (DCB: $\mathrm{n}=10$ vs. NCB: $\mathrm{n}=6$ ). Mean value of PD- $1 \mathrm{t} 1 / \mathrm{t} 0$ for DCB was 1.03 and that of $\mathrm{PD}-1 \mathrm{t} 1 / \mathrm{t} 0$ for NCB was 0.93 (P=0.76) (B) PFS stratified by PD $-1^{+}$CD8 ${ }^{+} \mathrm{T}$ cells t1/t0 (median PFS: 4.9 vs. 5.1 months, $\mathrm{P}=0.21$ ). (C) OS stratified by PD- $1^{+} \mathrm{CD} 8^{+} \mathrm{T}$ cells t1/t0 (median OS: 20.9 vs. 9.2 months, $\mathrm{P}=0.53)$. These experiments were repeated three times with similar results. PD-1, programmed cell death-1; PD-L1, programmed cell death ligand-1; DCB, durable clinical benefit; NCB, no clinical benefit; PBMCs, peripheral blood mononuclear cells; PFS, progression-free survival; OS, overall survival; t0, before immunotherapy; t1, after immunotherapy; ns, no significance.

comparison; Figure 2E).

\section{GrmB $t 1 / t 0$ as a potential adjunctive marker of PD-L1 TPS for immunotherapy}

Of the 16 patients included in the study, eight (50.0\%) demonstrated high PD-L1 TPS expression. Of the patients with strong PD-L1 staining, $75.0 \%$ displayed a DCB (Figure $3 A$ ). Evaluation of whether $\mathrm{GrmB} \mathrm{t} 1 / \mathrm{t} 0$ yielded a more accurate prediction when combined with the $\mathrm{PD}$ L1 level revealed three patients who manifested high PDL1 TPS ( $\geq 50 \%$ ), NCB, and low GrmB t1/t0 (patient IDs 1, 2, 11; Figure 3B). Conversely, only one patient with high GrmB t1/t0 and NCB showed a low (0\%) PD-L1 TPS. All patients who exhibited high $\mathrm{GrmB} \mathrm{t} 1 / \mathrm{t} 0$ and at least $1 \%$ PD-L1-expressing cancer cells in immunohistochemistry assays showed DCB (Figure 3B).

We analyzed the Kaplan-Meier curves to further understand the predictive performance of GrmB t1/ t0 and PD-L1 TPS $(\geq 1 \%)$. Our analysis indicated that patients with high $\mathrm{GrmB}$ t1/t0 sequential change $(>0.805)$ exhibited a longer median PFS and OS than patients with lower GrmB fold-changes $(<0.805)$ (PFS: 12.5 vs. 1.6 months; $\mathrm{P}=0.003$ \& OS: 21.0 vs. 7.4 months; $\mathrm{P}=0.003$ ) (Figure $3 C, 3 D$ ). We then calculated ICCs to evaluate the concordance of PD-L1 TPS and GrmB t1/t0. The ICC for PD-L1 TPS and GrmB t1/t0 expression was $0.22(\mathrm{P}=0.460)$, indicating poor agreement. Therefore, the combination of PD-L1 TPS—resulting in at least 1\% PD-L1-expressing cancer cells-and high $\mathrm{GrmB}$ t1/t0 appeared to possess optimum predictive power concerning immune-checkpoint inhibitor efficacy.

\section{Other immune markers correlated with immunotherapy response in NSCLC patients}

We also investigated the effects of immunotherapy on inhibitory molecules, including Tim-3, LAG-3, and CTLA-4; these molecules are associated with the exhausted phenotype, defined as a failure to proliferate (16). We determined that immunotherapy did not affect any of the inhibitory molecules (Figure $4 A$ ). Flow cytometry showed that $\mathrm{CD}^{+}{ }^{+} \mathrm{FoxP}^{+} \mathrm{T}$ cells occurred more frequently in patients with lung cancer than in healthy controls. However, like the inhibitory molecules, FoxP ${ }^{+} \mathrm{T}$ cells were not correlated with immunotherapy (Figure 4A).

After studying cytokines secreted from $\mathrm{CD} 8^{+} \mathrm{T}$ cells, we found that fold-changes of IL-2 and TNF- $\alpha$ concentration did not impact the clinical benefit or PFS at any time point (Figure 4B). Although patients with DCB had higher IFN- $\gamma$ $\mathrm{t} 1 / \mathrm{t} 0$ than patients with $\mathrm{NCB}$, this difference was not significant $(\mathrm{P}=0.058)$. Nevertheless, we analyzed IFN- $\gamma \mathrm{t} 1 /$ t0 because it relies on responses to anti-PD-1 antibodies. As a means to stratify responses to immunotherapy, IFN- $\gamma$ t1/ t0 exhibited an optimal index of 0.890 , with a sensitivity of $70 \%$ and a specificity of $67 \%$, obtained from the ROC curve (Figure 4C). Based on this optimal index, IFN- $\gamma \mathrm{t} 1 / \mathrm{t} 0$ level dichotomized patients into "high" and "low" subunits. The 
A

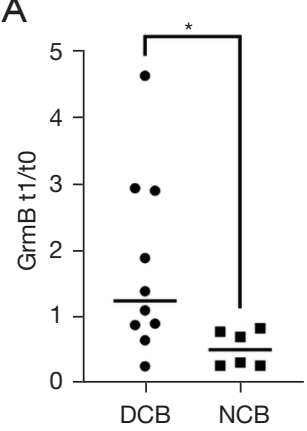

B

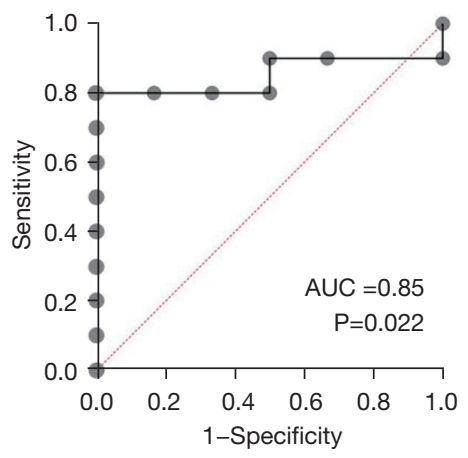

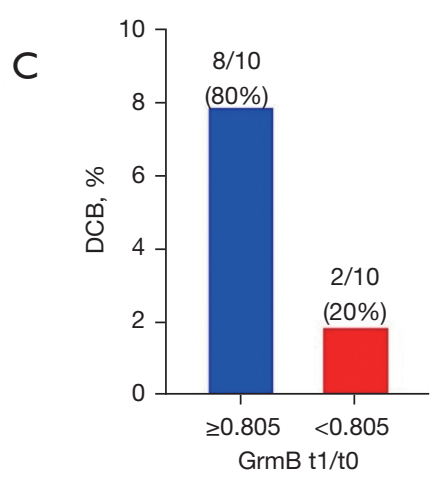

D

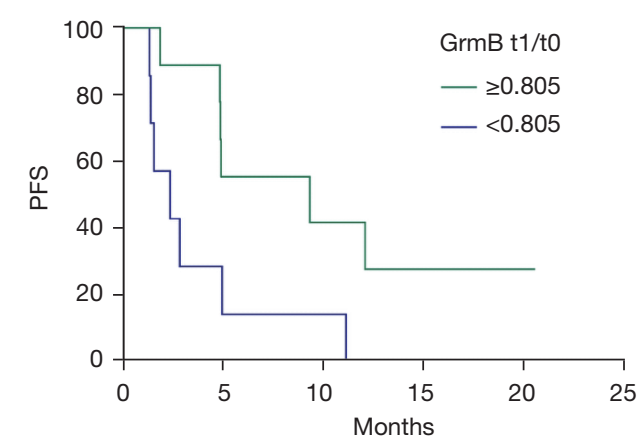

E

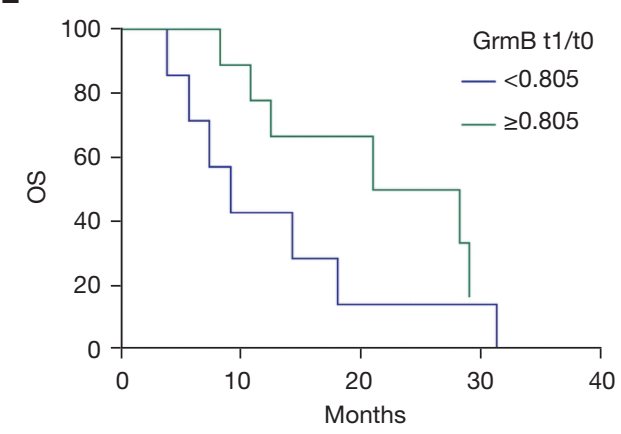

Figure 2 Correlations of the change in percentage of $\mathrm{PD}-1^{+} \mathrm{CD} 8^{+} \mathrm{T}$ cells expressing GrmB with the response and clinical outcomes associated with immune-checkpoint inhibitors. (A) Comparison of GrmB t1/t0 between DCB and NCB subgroups after immune-checkpoint inhibitor administration (DCB: $\mathrm{n}=10$ vs. NCB: $\mathrm{n}=6$ ). Mean value of GrmB t1/t0 for DCB was 1.75 and that of $\mathrm{GrmB} \mathrm{t} 1 / \mathrm{t} 0 \mathrm{for} \mathrm{NCB}$ was $0.52(\mathrm{P}=0.048)(\mathrm{B})$ The ROC curve and optimal cutoff value (0.805) for predicting patients with DCB $(\mathrm{P}=0.022)$. (C) Percentage of DCB in patients with $\mathrm{GrmB} t 1 / \mathrm{t} 0 \geq 0.805$ and $\mathrm{GrmB} \mathrm{t} 1 / \mathrm{t} 0<0.805$ ( $80 \%$ and 20\%, respectively). (D) Kaplan-Meier curves of PFS in patients with $\mathrm{GrmB} \mathrm{t} 1 / \mathrm{t} 0 \geq 0.805$ and $\mathrm{GrmB} \mathrm{t} 1 / \mathrm{t} 0<0.805$ showed 9.4 months and 2.4 months with statistical significance (P=0.025). (E) KaplanMeier curves of OS in patients with GrmB $\mathrm{t} 1 / \mathrm{t} 0 \geq 0.805$ and $\mathrm{GrmB} \mathrm{t} 1 / \mathrm{t} 0<0.805$ revealed 24.6 months and 9.2 months without significance $(\mathrm{P}=0.18)$. These experiments were repeated three times with similar results. ${ }^{*} \mathrm{P}<0.05$; ns, no significance; $\mathrm{PD}-1$, programmed cell death-1; DCB, durable clinical benefit; GrmB, granzyme B; NCB, no clinical benefit; ROC, receiver operating characteristic; PFS, progression-free survival; t0, before immunotherapy; $\mathrm{t} 1$, after immunotherapy.

probability of DCB was higher in patients with high IFN- $\gamma$ $\mathrm{t} 1 / \mathrm{t} 0$ than in patients with low IFN- $\gamma \mathrm{t} 1 / \mathrm{t} 0(70 \% v s .30 \%$; Figure 4D) Patients with high IFN- $\gamma \mathrm{t} 1 / \mathrm{t} 0$ had a longer PFS than those with low IFN- $\gamma \mathrm{t} 1 / \mathrm{t} 0$ (7.9 months for high IFN- $\gamma$ $\mathrm{t} 1 / \mathrm{t} 0$ vs. 3.8 months for low IFN- $\gamma \mathrm{t} 1 / \mathrm{t} 0)$, although the difference was not significant $(\mathrm{P}=0.215)$ (Figure $4 E$ ).

\section{Discussion}

PD-1/PD-L1 blockade inhibitors have demonstrated significant improvements in survival in patients with advanced NSCLC. However, the optimal biomarker for predicting the efficacy of PD-1/PD-L1 blockade inhibitors has not been identified beyond PD-L1 TPS that has several drawbacks. The discovery of biomarkers to accurately predict immunotherapy for lung cancer is crucial. This study found that the change in peripheral $\mathrm{PD}-1^{+} \mathrm{CD} 8^{+} \mathrm{T}$ cells expressing GrmB can predict immunotherapy efficacy in NSCLC patients.

We initially hypothesized that post-immunotherapy peripheral $\mathrm{CD}^{+} \mathrm{T}$-cell responses reflect the activation of tumor-specific PD- $1^{+} \mathrm{CD} 8^{+} \mathrm{T}$ cells. However, we observed that proliferative changes in peripheral $\mathrm{PD}-1^{+} \mathrm{CD} 8^{+} \mathrm{T}$ cells $(\mathrm{t} 1 / \mathrm{t} 0)$ did not significantly affect responses to therapy. In contrast to our data, recent studies have revealed positive results regarding the use of peripheral $\mathrm{PD}-1^{+} \mathrm{T}$ cells as 

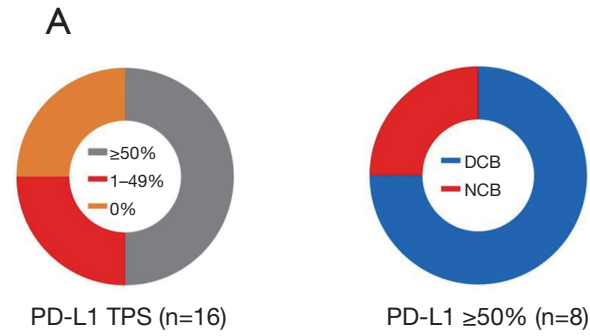

PD-L1 $\geq 50 \%(n=8)$

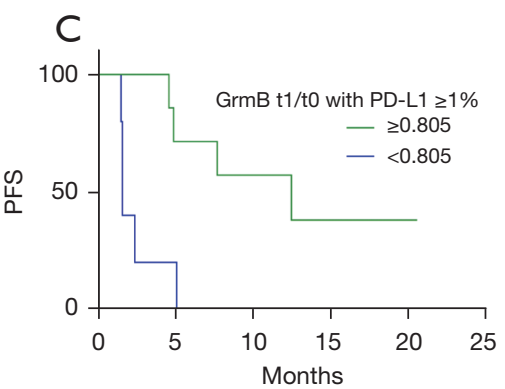

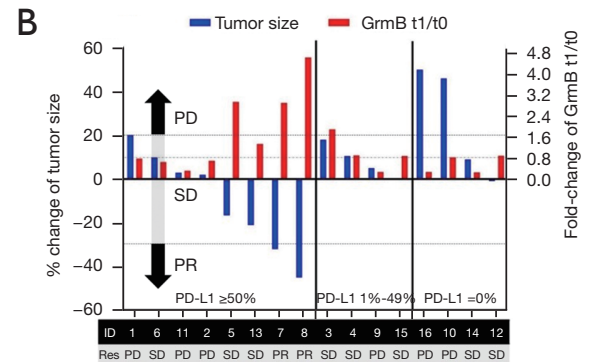

D

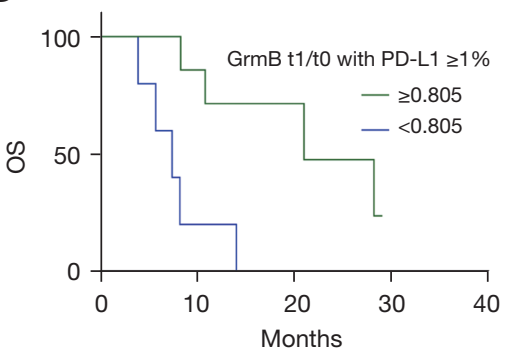

Figure $3 \mathrm{GrmB}$ t1/t0 and intratumoral PD-L1 TPS level are associated with response. (A) Graphs show the frequency of different clinical outcomes according to PD-L1 TPS ( $\mathrm{n}=16)$. (B) Waterfall plot of the observed response to immunotherapy and corresponding changes in the GrmB t1/t0 and PD-L1 TPS level. The left y-axis represents the change in tumor size, PD means when the tumor size increased by more than 20\% (upper dotted line), PR reduced by more than 30\% (lower dotted line), SD means the change between PD and PR. The middle dotted line indicates the cut-off value (0.805) of GrmB t1/t0 based on the right $y$-axis indicating GrmB t1/t0. (C) Kaplan-Meier curves of PFS in patients with GrmB $\mathrm{t} 1 / \mathrm{t} 0 \geq 0.805$ and GrmB $\mathrm{t} 1 / \mathrm{t} 0<0.805$ as well as PD-L1 TPS ( $\geq 1 \%$ ). (D) Kaplan-Meier curves of OS in patients with GrmB t1/t0 $\geq 0.805$ and GrmB t1/t0 <0.805 as well as PD-L1 TPS ( $\geq 1 \%)$. PD-L1, programmed cell death ligand-1; GrmB, granzyme B; TPS, tumor proportion score; PFS, progression-free survival; t0, before immunotherapy; $\mathrm{t}$, after immunotherapy; PD, progressive disease; $\mathrm{SD}$, stable disease; $\mathrm{PR}$, partial response.

predictors of immunotherapy outcomes. For example, Kim et al. showed that the first-week proliferation of peripheral blood PD- $1^{+} \mathrm{CD}^{+} \mathrm{T}$ cells obtained from 79 patients with NSCLC predicts the response to anti-PD-1 therapy in solid tumors (13). Another study indicated that PD $-1^{+} \mathrm{CD} 8^{+} \mathrm{T}$ cells were activated and detectable in peripheral blood of 29 NSCLC patients within three weeks (14). Our discrepant results to detect $\mathrm{PD}-1^{+} \mathrm{CD}{ }^{+} \mathrm{T}$-cell responses may have been because of sample size. Therefore, the optimal number of blood sampling warrants further study to validate the possibility of $\mathrm{PD}-1^{+} \mathrm{CD}^{+} \mathrm{T}$ cells predicting therapeutic response.

Furthermore, we evaluated the percentage change in proliferating $\mathrm{PD}-1^{+} \mathrm{CD} 8^{+} \mathrm{T}$ cells expressing $\mathrm{GrmB}$ and its clinical relevance regarding immunotherapy. A previous study revealed that effector $\mathrm{T}$ cells deficient in GrmB eliminate targets much slower than do wild-type cells. This underscores the protease's vital role in effecting the timely demise of tumorigenic cells associated with immunotherapy (17). Our results indicated that the GrmB $\mathrm{t} 1 / \mathrm{t} 0$ level affected both the immunotherapy response and PFS rate. Although GrmB is widely known as an activator and marker of $\mathrm{T}$ cells, relatively few studies have analyzed GrmB in detail. Here, we measured the postimmunotherapy proportion of live $\mathrm{T}$ cells producing GrmB after immunotherapy and the pre-immunotherapy proportion of $\mathrm{GrmB}^{+} \mathrm{PD}-1^{+} \mathrm{CD}^{+} \mathrm{T}$ cells. These measurements are essential because cytotoxic $\mathrm{T}$ cells also secrete different cytokines and perforins. We determined the proportion of $\mathrm{PD}-1^{+} \mathrm{CD} 8^{+} \mathrm{T}$ cells that solely produced GrmB after immunotherapy by dividing these values. Within the first four weeks after treatment initiation, $\mathrm{CD} 8^{+}$ T-cell responses are reportedly triggered by blocking the PD-1 pathway and may be associated with favorable clinical outcomes (14). Our data highlight that treatmentrelated changes in $\mathrm{GrmB} t 1 / \mathrm{t} 0$ were sustained even at three weeks after treatment initiation, despite inconsistent differences in PD- $1^{+} \mathrm{T}$ cell percentages. Therefore, GrmB $\mathrm{t} 1 / \mathrm{t} 0$ is a potential predictive biomarker for investigating immunotherapy response. 

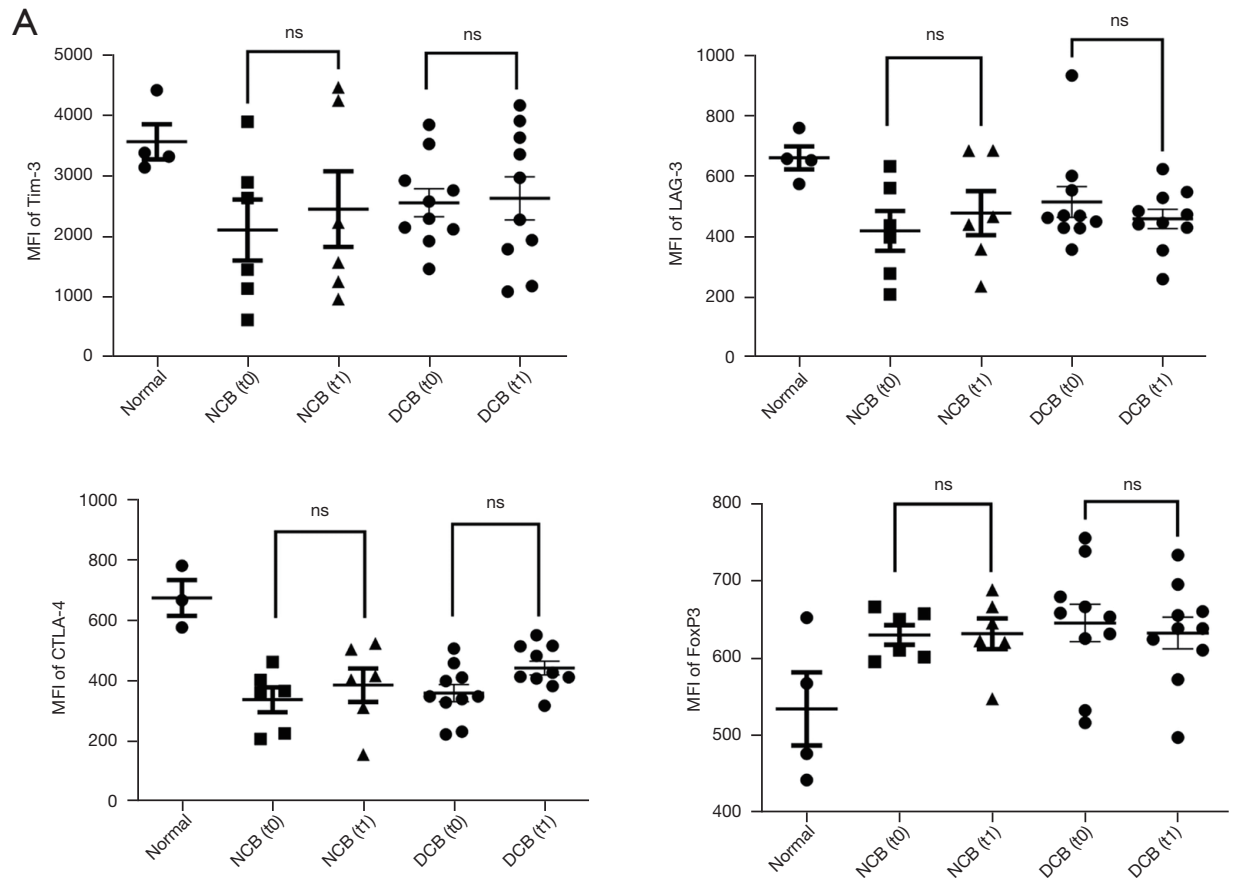

B

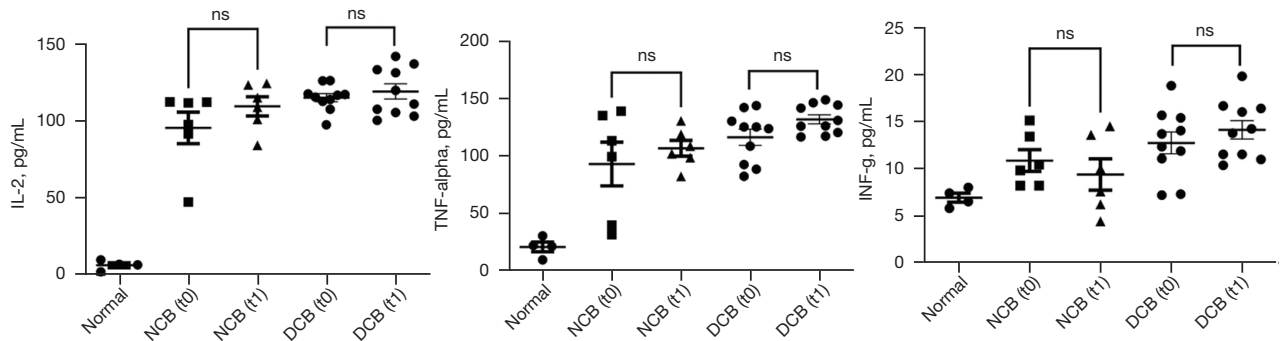

C

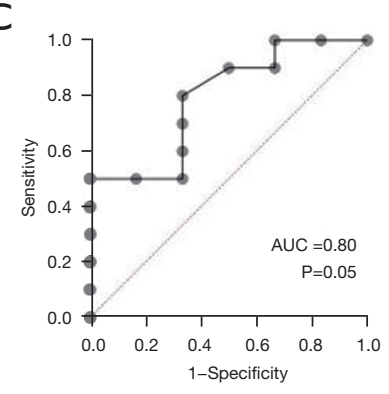

D

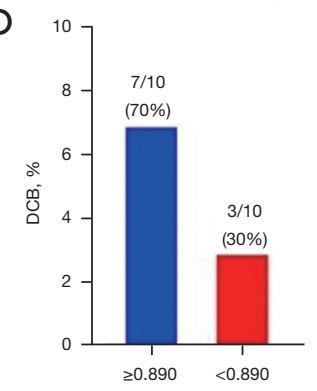

E

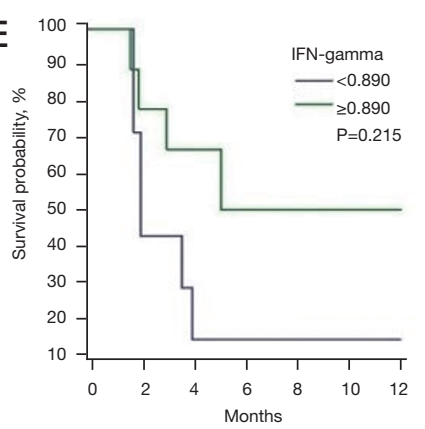

Figure 4 Co-receptor expression and functionality of peripheral blood CD8 ${ }^{+} \mathrm{T}$ cells of NSCLC patients treated with immune-checkpoint inhibitors. (A) Tim-3, LAG-3, and CTLA-4 mean fluorescence intensity on $\mathrm{CD}^{+} \mathrm{T}$ cells $(\mathrm{n}=16)$ and $\mathrm{FoxP}^{+} \mathrm{T}$ cells ( $\left.\mathrm{n}=16\right)$. (B) Effector cytokine secretion of sorted $\mathrm{CD} 8^{+} \mathrm{T}$ cells with immunotherapy response ( $\left.\mathrm{n}=16\right)$. (C) The ROC curve and optimal cutoff value (0.890) for predicting patients with DCB ( $\mathrm{n}=16$ ). (D) Percentage DCB in patients with IFN- $\gamma \mathrm{t} 1 / \mathrm{t} 0 \geq 0.890$ and IFN- $\gamma \mathrm{t} 1 / \mathrm{t} 0<0.890$. (E) Kaplan-Meier curves of PFS in patients with IFN- $\gamma \mathrm{t} 1 / \mathrm{t} 0 \geq 0.890$ and IFN- $\gamma \mathrm{t} 1 / \mathrm{t} 0<0.890$. These experiments were repeated three times with similar results. ns, no significance; FoxP3, Forkhead box protein P3; Tim-3, T cell immunoglobulin and mucin domain-containing-3; LAG-3, lymphocyte activation gene-3; CTLA-4, cytotoxic T lymphocyte antigen-4; IL-2, interleukin-2; TNF $\alpha$, tumor necrosis factor-alpha; IFN- $\gamma$, interferongamma; DCB, durable clinical benefit; ROC, receiver operating characteristic; PFS, progression-free survival; $\mathrm{t} 0$, before immunotherapy; $\mathrm{t}$, after immunotherapy; NSCLC, non-small cell lung cancer 
Because the study design included patients treated with any types of PD-1/PD-L1 inhibitors and prospective blood collection, we did not control patients treated with various therapeutic drugs. We postulated that both PD-1 and PDL1 inhibitors would show the exact cytotoxic mechanisms associated with GrmB since immune cells secrete GrmB in response to PD-1/PD-L1 blockade. Previous studies $(18,19)$ support this idea, with increased expression of GrmB on $\mathrm{CD}^{+} \mathrm{T}$ cells in solid tumors associated with worse clinical outcomes in the response group to different types of PD-L1 inhibitors. Moreover, although the relationship between atezolizumab and GrmB was not directly shown, a subgroup of previous studies analyzing the side effects of immunotherapy confirmed that atezolizumab increased tumor infiltrate lymphocytes and increased GrmB staining (20). Therefore, our results suggested the potential role of GrmB regarding the efficacy of various immunotherapeutic agents.

We calculated the concordance between available PD$\mathrm{L} 1$ and $\mathrm{GrmB}$ t1/t0 values to evaluate whether GrmB t1/ t0 is a suitable alternative index. However, we found poor agreement, indicating that $\mathrm{GrmB} \mathrm{t} 1 / \mathrm{t} 0$ could not replace $\mathrm{PD}$ $\mathrm{L} 1$ as a predictive marker. However, the combination of $\mathrm{PD}$ L1 TPS ( $\geq 1 \%)$ and high GrmB $\mathrm{t} 1 / \mathrm{t} 0$ had better predictive potential than any single parameter. Therefore, our study suggests a possible role for $\mathrm{GrmB} \mathrm{t} 1 / \mathrm{t} 0$ as an adjunctive predictive biomarker of immunotherapy with PD-L1 TPS.

Because targeting the PD-1- or PD-L1-related pathways does not entirely restore $T$ cell function (21), we identified inhibitory pathways involved in T cell exhaustion. FoxP3 is a transcription factor specific to regulatory $\mathrm{T}$ lymphocytes. FoxP $3^{+}$regulatory $\mathrm{T}$ cells exert their immunosuppressive effects through various mechanisms, including the production of immune-inhibitory cytokines (22). High levels of tumor-infiltrating FoxP3 ${ }^{+}$regulatory $\mathrm{T}$ cells are an unfavorable prognostic factor for overall survival (23). Here, we found that patients with NSCLC had more FoxP3 ${ }^{+}$ $\mathrm{T}$ cells than the healthy population (Figure 4). FoxP3 ${ }^{+} \mathrm{T}$ cell count in blood was not significantly associated with an immunotherapy response despite decreasing after immunotherapy. Additionally, the expression of inhibitory molecules (LAG-3, TIM-3, and CTLA-4) decreased in peripheral blood $\mathrm{CD}^{+} \mathrm{T}$ cells. However, immunotherapy did not affect these inhibitory molecules, whose expression is associated with the manifestation of an exhausted phenotype and the production of IL-2, TNF- $\alpha$, and IFN- $\gamma$.

This study had several limitations. First, we sampled a small number of patients, limiting the statistical power of this study. Our research was terminated because of its small accrual of eligible patients who satisfied DCB criteria. We included patients with SD ( $>4.5$ months), which is lesser than the generally used SD criterion ( $>6$ months). Recent clinical trials showed that the median PFS of current immunotherapies was less than 4 months in patients with $\mathrm{PD}-\mathrm{L} 1>1 \%(24,25)$. As a parallel of these results, most patients in our institute seem to progress in about four months. However, we think this study still has a valuable meaning for the readers to potentiate a role of sequential GrmB as a complementary marker for patients with at least more duration than the known median PFS of immunotherapies. Thus, while these results indicate that GrmB may be a useful biomarker, more extensive studies are required to strengthen or refute our conclusions. We hope our study may inspire others to pursue larger data sets to investigate this area of great need-predicting responses to immune checkpoint inhibition. Second, immunotherapy efficacy can only be predicted after the first dose of antiPD-1 antibodies is given. Additionally, there was potential for human error during flow cytometry analysis. Despite these limitations, our preliminary findings warrant further studies analyzing peripheral blood $\mathrm{GrmB} \mathrm{t} 1 / \mathrm{t} 0$ in larger cohorts of lung cancer patients receiving immunecheckpoint inhibitor treatments.

In conclusion, sequential change in GrmB might be utilized as a predictive biomarker of immune checkpoint inhibitor monotherapy in advanced NSCLC.

\section{Acknowledgments}

We thank the Biobank of Pusan National University Yangsan Hospital for the provision of the biospecimens and data used for this study (No. OF-2018-03).

Funding: This work was supported by the Research Institute for Convergence of Biomedical Science and Technology, Pusan National University Yangsan Hospital (No. 302020-001) and a National Research Foundation of Korea grant, funded by the Korean government, MSIT (No. 2020R1F1A1065278).

\section{Footnote}

Reporting Checklist: The authors have completed the REMARK reporting checklist. Available at https://tcr. amegroups.com/article/view/10.21037/tcr-21-2506/rc

Data Sharing Statement: Available at https://tcr.amegroups. 
com/article/view/10.21037/tcr-21-2506/dss

Conflicts of Interest: All authors have completed the ICMJE uniform disclosure form (available at https://tcr.amegroups. com/article/view/10.21037/tcr-21-2506/coif). The authors have no conflicts of interest to declare.

Ethical Statement: The authors are accountable for all aspects of the work in ensuring that questions related to the accuracy or integrity of any part of the work are appropriately investigated and resolved. The study was conducted in accordance with the Declaration of Helsinki (as revised in 2013). It was approved by the Institutional Review Board of Pusan National University Yangsan Hospital (IRB 05-2018-061) and informed consent was taken from all the patients.

Open Access Statement: This is an Open Access article distributed in accordance with the Creative Commons Attribution-NonCommercial-NoDerivs 4.0 International License (CC BY-NC-ND 4.0), which permits the noncommercial replication and distribution of the article with the strict proviso that no changes or edits are made and the original work is properly cited (including links to both the formal publication through the relevant DOI and the license). See: https://creativecommons.org/licenses/by-nc-nd/4.0/.

\section{References}

1. Siegel RL, Miller KD, Jemal A. Cancer statistics, 2019. CA Cancer J Clin 2019;69:7-34.

2. Dawe DE, Harlos CH, Juergens RA. Immuno-oncologythe new paradigm of lung cancer treatment. Curr Oncol 2020;27:S78-86.

3. Incorvaia L, Fanale D, Badalamenti G, et al. Programmed Death Ligand 1 (PD-L1) as a Predictive Biomarker for Pembrolizumab Therapy in Patients with Advanced Non-Small-Cell Lung Cancer (NSCLC). Adv Ther 2019;36:2600-17.

4. Pirker R. Biomarkers for immune checkpoint inhibitors in advanced nonsmall cell lung cancer. Curr Opin Oncol 2019;31:24-8.

5. Brody R, Zhang Y, Ballas M, et al. PD-L1 expression in advanced NSCLC: Insights into risk stratification and treatment selection from a systematic literature review. Lung Cancer 2017;112:200-15.

6. Reck M, Rodríguez-Abreu D, Robinson AG, et al. Pembrolizumab versus Chemotherapy for PD-L1-
Positive Non-Small-Cell Lung Cancer. N Engl J Med 2016;375:1823-33.

7. von Pawel J, Bordoni R, Satouchi M, et al. Long-term survival in patients with advanced non-small-cell lung cancer treated with atezolizumab versus docetaxel: Results from the randomised phase III OAK study. Eur J Cancer 2019;107:124-32.

8. Chae YK, Pan A, Davis AA, et al. Biomarkers for PD-1/ PD-L1 Blockade Therapy in Non-Small-cell Lung Cancer: Is PD-L1 Expression a Good Marker for Patient Selection? Clin Lung Cancer 2016;17:350-61.

9. Xu Y, Wan B, Chen X, et al. The association of PDL1 expression with the efficacy of anti-PD-1/PD-L1 immunotherapy and survival of non-small cell lung cancer patients: a meta-analysis of randomized controlled trials. Transl Lung Cancer Res 2019;8:413-28.

10. Kim CG, Kim KH, Pyo KH, et al. Hyperprogressive disease during PD-1/PD-L1 blockade in patients with non-small-cell lung cancer. Ann Oncol 2019;30:1104-13.

11. Wojas-Krawczyk K, Kalinka E, Grenda A, et al. Beyond PD-L1 Markers for Lung Cancer Immunotherapy. Int J Mol Sci 2019;20:1915.

12. Hendriks LE, Rouleau E, Besse B. Clinical utility of tumor mutational burden in patients with non-small cell lung cancer treated with immunotherapy. Transl Lung Cancer Res 2018;7:647-60.

13. Kim KH, Cho J, Ku BM, et al. The First-week Proliferative Response of Peripheral Blood PD-1+CD8+ T Cells Predicts the Response to Anti-PD-1 Therapy in Solid Tumors. Clin Cancer Res 2019;25:2144-54.

14. Kamphorst AO, Pillai RN, Yang S, et al. Proliferation of PD-1+ CD8 T cells in peripheral blood after PD-1targeted therapy in lung cancer patients. Proc Natl Acad Sci U S A 2017;114:4993-8.

15. Hodge G, Barnawi J, Jurisevic C, et al. Lung cancer is associated with decreased expression of perforin, granzyme $\mathrm{B}$ and interferon (IFN)- $\gamma$ by infiltrating lung tissue T cells, natural killer (NK) T-like and NK cells. Clin Exp Immunol 2014;178:79-85.

16. Larimer BM, Wehrenberg-Klee E, Dubois F, et al. Granzyme B PET Imaging as a Predictive Biomarker of Immunotherapy Response. Cancer Res 2017;77:2318-27.

17. Pardo J, Bosque A, Brehm R, et al. Apoptotic pathways are selectively activated by granzyme A and/or granzyme $\mathrm{B}$ in CTL-mediated target cell lysis. J Cell Biol 2004;167:457-68.

18. Tumeh PC, Harview Cl, Yerley JH, et al. PD-1 blockade induceds responses by inhibiting adaptive immune 
resistance. Nature 2014;515:568-71.

19. Hurkmans DP, Basak EA, Schepers N, et al. Granzyme B is correlated with clinical outcome after PD-1 blockade in patients with stage IV non-small-cell lung cancer. J Immunother Cancer 2020;8:e000586.

20. Sobol I, Chen CL, Mahmood SS, et al. Histopathologic Characterization of Myocarditis Associated With Immune Checkpoint Inhibitor Therapy. Arch Pathol Lab Med 2020;144:1392-6.

21. Rossi G, Russo A, Tagliamento M, et al. Precision Medicine for NSCLC in the Era of Immunotherapy: New Biomarkers to Select the Most Suitable Treatment or the Most Suitable Patient. Cancers (Basel) 2020;12:1125.

22. Blackburn SD, Shin H, Freeman GJ, et al. Selective expansion of a subset of exhausted CD8 T cells by

Cite this article as: Chung JH, Ha JS, Choi J, Kwon SM, Yun MS, Kim T, Jeon D, Yoon SH, Kim YS. Granzyme B for predicting the durable clinical benefit of anti-PD-1/PD-L1 immunotherapy in patients with non-small cell lung cancer. Transl Cancer Res 2022;11(2):316-326. doi: 10.21037/tcr-21-2506
alphaPD-L1 blockade. Proc Natl Acad Sci U S A 2008;105:15016-21.

23. Shang B, Liu Y, Jiang SJ, et al. Prognostic value of tumorinfiltrating FoxP3 + regulatory T cells in cancers: a systematic review and meta-analysis. Sci Rep 2015;5:15179.

24. Herbst RS, Garon EB, Kim DW, et al. Five Year Survival Update From KEYNOTE-010: Pembrolizumab Versus Docetaxel for Previously Treated, Programmed DeathLigand 1-Positive Advanced NSCLC. J Thorac Oncol 2021;16:1718-32.

25. Borghaei H, Gettinger S, Vokes EE, et al. Five-Year Outcomes From the Randomized, Phase III Trials CheckMate 017 and 057: Nivolumab Versus Docetaxel in Previously Treated Non-Small-Cell Lung Cancer. J Clin Oncol 2021;39:723-33. 

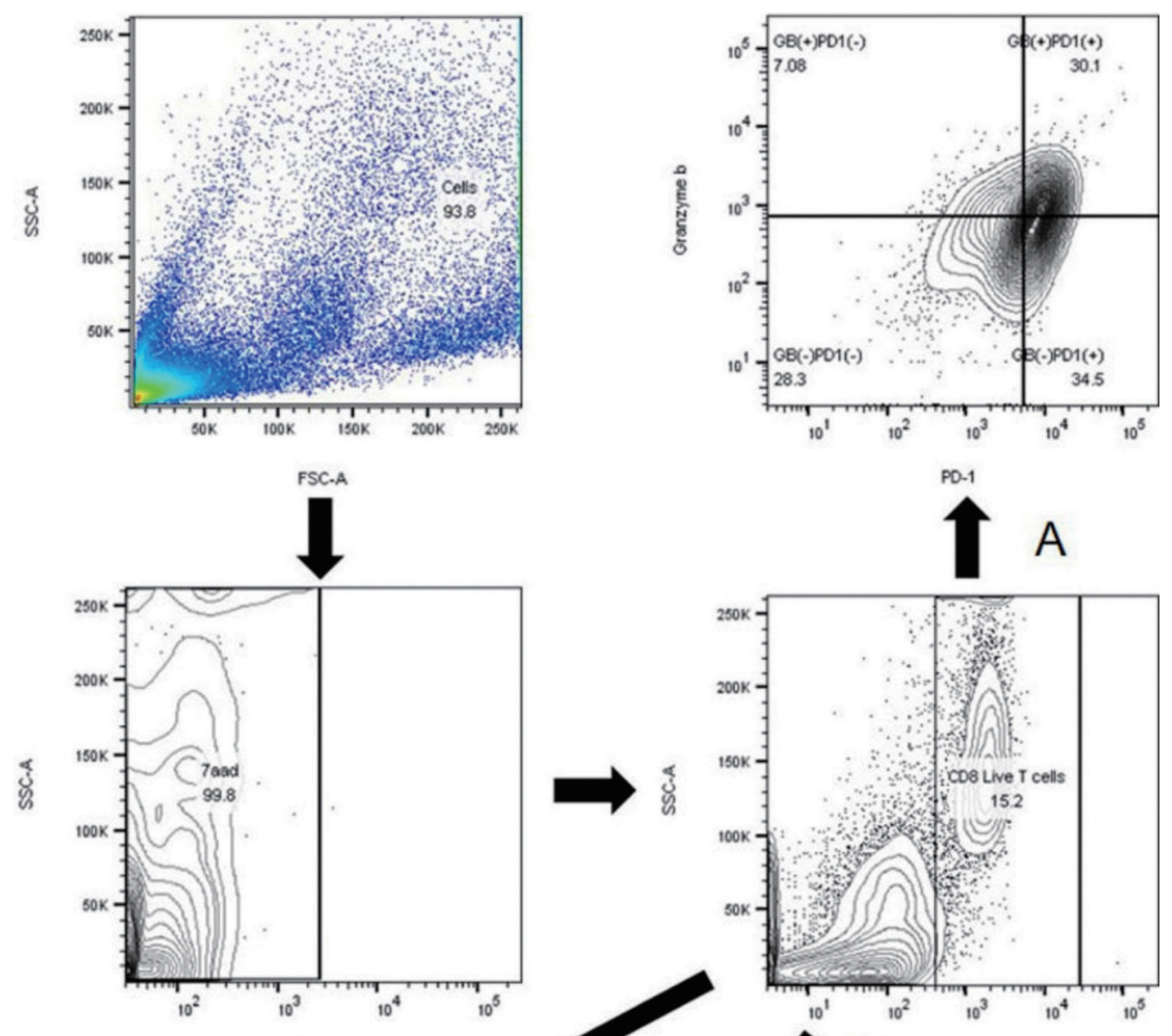

Live cel
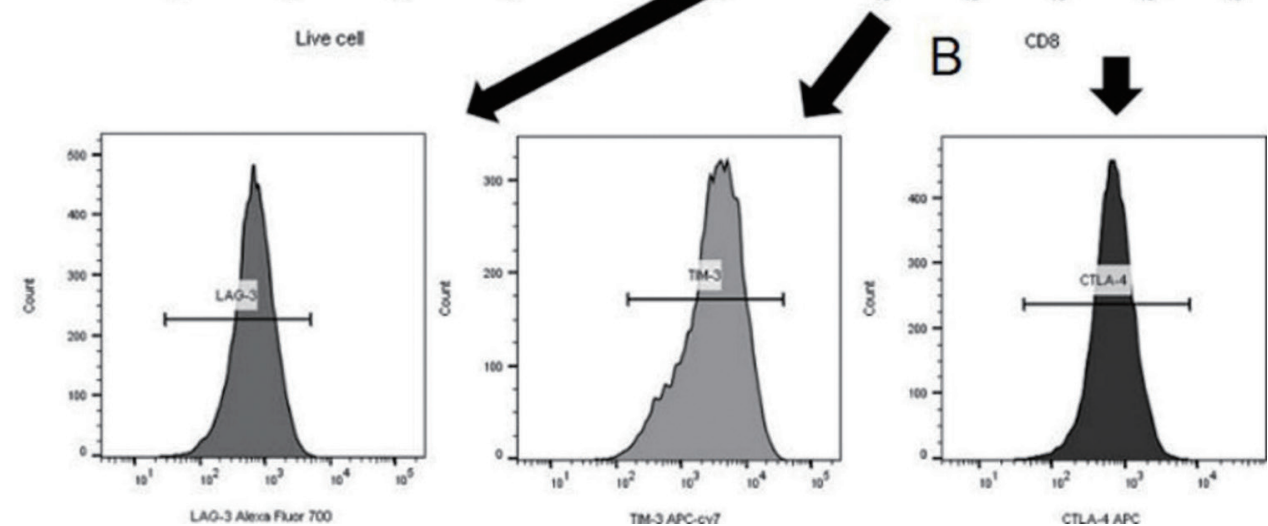

Figure S1 Gating strategy for flow cytometric analysis. (A) Gating strategy for PD-1 $1^{+} \mathrm{CD} 8^{+} \mathrm{T}$ cells and (B) co-receptor expression. PD-1, programmed cell death-1; GB, granzyme B; SSC-A, Side Scatter Area; FSC-A, forward scatter area; 7aad, 7-amino actinomycin D.
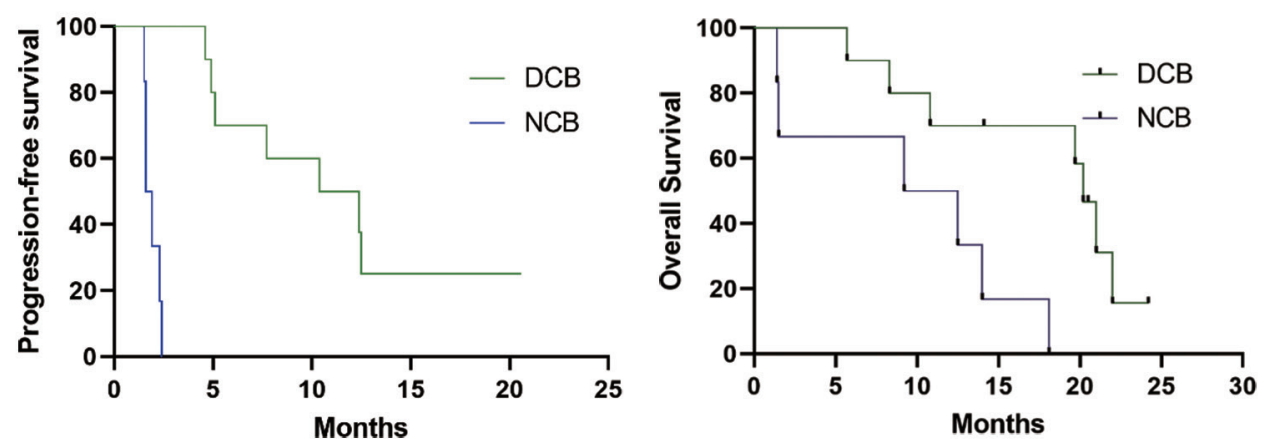

Figure S2 Progression-free survival and overall survival of NSCLC patients. The median PFS of DCB was 11.40 months, and that of NCB was 1.75 months $(\mathrm{P}<0.0001)$. The median OS of DCB was 28.20 months, and that of NCB was 10.85 months. The hazard ratio (95\% confidence interval of ratio) for OS was $0.16(0.03-0.84)$ for patients regardless of PD-L1 TPS expression. The survival curves are significantly different with $\mathrm{P}=0.013$. $\mathrm{DCB}$, durable clinical benefit; $\mathrm{NCB}$, no clinical benefit. 


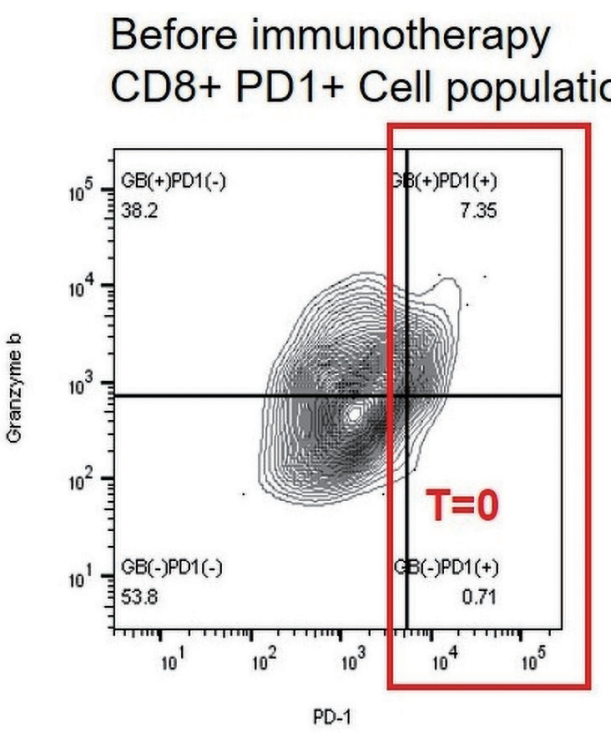

Specimen_001_9-1_018.fcs CD8 Live T cells 422
After immunotherapy CD8+ PD1+ Cell population

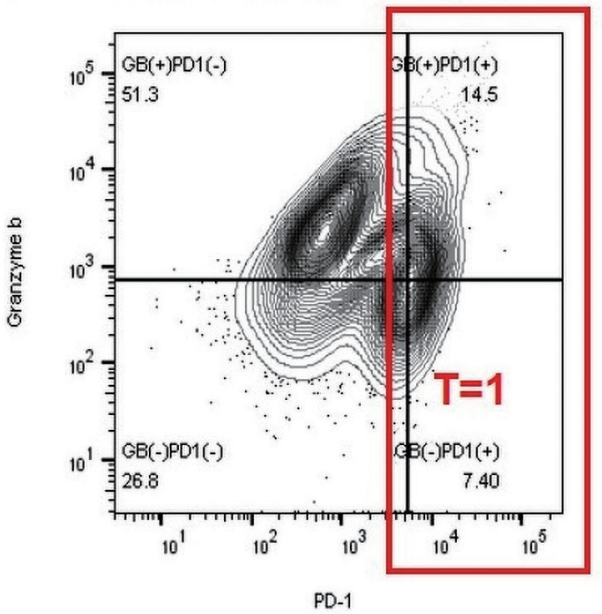

Specimen_001_9-2_019.fcs CD8 Live T cells 9955

Figure S3 Representative images of FACS analysis on peripheral blood mononuclear cells (PBMCs) from patients before and after treatment with immune check point inhibitors. Flow cytometry images of the PD- $1^{+} \mathrm{CD} 8^{+}$T-cell population. PD-1, programmed cell death-1; GB, granzyme B.

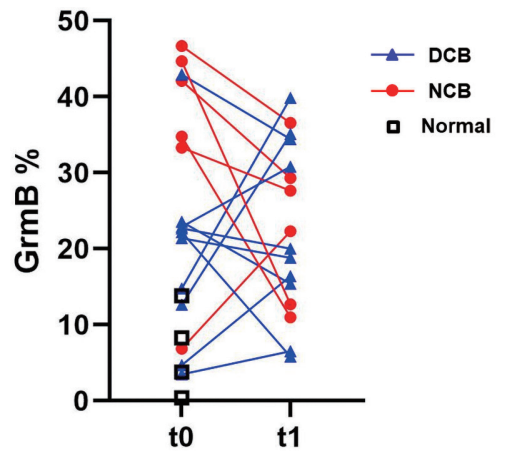

Figure S4 Proliferative response of $\mathrm{PD}-1^{+} \mathrm{CD} 8^{+} \mathrm{T}$ cells containing GrmB in peripheral blood collected before ( $\mathrm{t} 0$ ) and after anti-PD-1 therapy (t1). Changes in percentage of PD $-1^{+} \mathrm{CD}^{+} \mathrm{T}$ cells containing GrmB from patients with normal patients $(\mathrm{n}=4)$ and lung cancer $(\mathrm{n}=16)$ before and after immune check point inhibitors. DCB, durable clinical benefit; NCB, no clinical benefit; GrmB, granzyme B; t0, before immunotherapy; t1, after immunotherapy. 
Table S1 Patient's clinicopathological characteristics

\begin{tabular}{|c|c|c|c|c|c|c|c|c|}
\hline Patient ID & Age/Sex & Histology & Smoking status & TNM stage & $\mathrm{PD}-\mathrm{L} 1^{\mathrm{a}}$ & $\mathrm{ICl}$ & Time to sampling (days) & Response \\
\hline 1 & $77 / M$ & SQC & Ex-smoker & IV & 2 & Nivo & 21 & PD \\
\hline 2 & $68 / M$ & SQC & Current smoker & IV & 2 & Nivo & 18 & PD \\
\hline 3 & $71 / \mathrm{M}$ & SQC & Current smoker & IV & 1 & Nivo & 18 & SD \\
\hline 4 & $50 / M$ & SQC & Current smoker & IV & 1 & Nivo & 17 & SD \\
\hline 5 & $68 / M$ & SQC & Current smoker & IV & 2 & Pembro & 21 & SD \\
\hline 6 & $85 / M$ & SQC & Ex-smoker & IIIc & 2 & Pembro & 21 & SD \\
\hline 7 & $81 / \mathrm{M}$ & SQC & Ex-smoker & IV & 2 & Pembro & 22 & PR \\
\hline 8 & $61 / M$ & $A D C$ & Ex-smoker & IV & 2 & Pembro & 21 & PR \\
\hline 9 & $46 / M$ & $A D C$ & Current smoker & IV & 1 & Nivo & 18 & PD \\
\hline 10 & $75 / M$ & $A D C$ & Current smoker & IV & 2 & Atezo & 21 & PD \\
\hline 11 & $58 / \mathrm{M}$ & SQC & Ex-smoker & IV & 0 & Pembro & 21 & PD \\
\hline 12 & $79 / M$ & SQC & Ex-smoker & IIIc & 2 & Atezo & 21 & $S D^{b}$ \\
\hline 13 & $79 / M$ & $A D C$ & Current smoker & IV & 2 & Pembro & 20 & SD \\
\hline 14 & $73 / M$ & SQC & Current smoker & IV & 0 & Atezo & 25 & SD \\
\hline 15 & $67 / M$ & $A D C$ & Ex-smoker & IV & 1 & Atezo & 25 & SD \\
\hline 16 & $87 / \mathrm{M}$ & $A D C$ & Ex-smoker & IIIC & 0 & Atezo & 21 & PD \\
\hline
\end{tabular}

a PD-L1 expression (SP263, TPS): 0\%: 0 vs. 1-49\%: 1 vs. $\geq 50 \%$ : 2. SQC: squamous cell carcinoma; ADC: adenocarcinoma; PD: progressive disease; SD: stable disease; PR: partial response; ICl: immune checkpoint inhibitors; Nivo: nivolumab; Pembro: pembrolizumab; Atezo: atezolizumab.

Table S2 Antibody information

\begin{tabular}{lll}
\hline Antibody & Source & Catalog No \\
\hline Anti-Mouse CD8a (Clone: 5.3-6.7) & Biolegend & 100710 \\
Anti-Human/Mouse Granzyme B (Clone: QA16A02) & Biolegend & 372214 \\
Anti-Mouse CD279 (PD-1) (Clone: J43) & eBiosciences & $12-9985-82$ \\
Anti-Human FoxP3 (Clone: PCH101) & eBiosciences & $11-4776-42$ \\
Anti-Human CD152 (CTLA-4) (Clone: L3D10) & Biolegend & 349908 \\
Anti-Human CD223 (LAG-3) (Clone: 3DS223H) & eBiosciences & $56-2239-42$ \\
Anti-Human CD336 (Tim-3) (Clone: F38-2E2) & Biolegend & 345026 \\
7-AAD staining solution & Abcam & ab228563 \\
\hline
\end{tabular}

